\section{Audiometria de alta freqüência: estudo com indivíduos audiologicamente normais}

\author{
Daniela R. Sahyeb ${ }^{1}$, Orozimbo A. Costa Filho', \\ Kátia de F. Alvarenga ${ }^{3}$
}

\section{High-frequency audiometry: study with normal audiological subjects}

Resumo / Summary

$\mathbf{P}$ esquisas recentes apontam a Audiometria Tonal de Alta Freqüência (AT-AF) como um instrumento para o diagnóstico precoce de danos auditivos decorrentes de alguns agentes etiológicos principais, como envelhecimento e exposição a drogas ototóxicas e a intensidades elevadas de ruído. Objetivo: Apesar de já existirem várias técnicas desenvolvidas para essa avaliação, algumas não se aplicam à rotina clínica, em função da falta de praticidade e, por vezes, falta de consistência nos resultados. Segundo a literatura, ainda estão por emergir uma metodologia adequada a tal avaliação e valores indicados como referência à normalidade. Forma de Estudo: Clínico prospectivo. Material e método: A presente pesquisa observou o comportamento dos limiares auditivos de alta freqüência em indivíduos jovens e audiologicamente normais e analisou variabilidades acústicas, inter e intra-indivíduos, que de acordo com a literatura, podem interferir na estabilidade dos resultados. Conclusão: Com os dados obtidos, pôde-se estabelecer valores de média, desvio padrão e mediana, além de valores mínimos e máximos para cada freqüência. Os testes estatísticos não identificaram diferenças significantes na maioria das análises realizadas (entre sexo, interaurais, variabilidades acústicas e intra-indivíduos, no mesmo dia de testes). A variabilidade dos resultados entre os exames de um mesmo indivíduo, realizados em dias deferentes de testes, mostrou ser significante, sendo as médias dos limiares no segundo dia sempre melhores que as do primeiro dia.
Palavras-chave: audiologia, audiometria. Key words: audiometry, audiology.
$\mathbf{R}$ ecent research studies pointed to High-Frequency Audiometry (HFA) as a tool for early diagnosis of hearing impairment caused by the main etiological agents, such as aging, exposure to ototoxic drugs, and occupational noise. Aim: Although there are already several techniques developed for this assessment, some of them should not be applied to clinical routine, because of their lack of practicability and, sometimes, lack of reliable results. According to the literature, a suitable methodology for such assessment, as well as values indicated as normal reference, are still needed. Study design: Clinical prospective. Material and method: The present research study observed auditory thresholds in young and audiologically normal individual with HFA and assessed some variables that could have an influence upon the results. Conclusion: Based on the collected data, we were able to established values for mean, standard deviation and median, besides minimum and maximum values for each frequency, for the entire population. Statistical analyses did not identify significant differences between gender and the ears. Moreover, questions related to inter-and intra-individual variability are discussed.

\footnotetext{
${ }^{1}$ Fonoaudióloga do Centro de Atendimentos de Distúrbios da Audição, Linguagem e Visão do Hospital de Reabilitação de Anomalias Craniofaciais da Universidade de São Paulo (CEDALVI - HRAC - USP) e Mestre em Distúrbios da Comunicação pela Pontifícia Universidade Católica de São Paulo.

${ }^{2}$ Médico do Hospital de Reabilitação de Anomalias Craniofaciais da Universidade de São Paulo (HRAC-USP) e do Departamento de Fonoaudiologia da Faculdade de Odontologia de Bauru (FOB-USP), responsável pelo Centro de Pesquisas Audiológicas do Hospital de Reabilitação de Anomalias Craniofaciais (CPA-HRAC-USP). ${ }^{3}$ Professora Doutora do Curso de Fonoaudiologia da Faculdade de Odontologia de Bauru da Universidade de São Paulo (FOB-USP). Instituição: Pontifícia Universidade Católica de São Paulo Endereço para correspondência: Daniela Rodrigues Shayeb - R. Júlio de Mesquita Filho 6-27 Vila Universitária. Bauru SP 17011-450 Tel (0xx14) 234-1335 ou 234-3563 e 234-7884, ramal 220 - E-mail: nancishayeb@uol.com.br Órgão fomentador da pesquisa: Capes.

Pesquisa aprovada pelo Comitê de Ética em Pesquisa do HRAC-USP, sob protocolo no 93/2002-UEP-PC.

Dissertação de Mestrado apresentada como Conferência no $15^{\circ}$ Encontro Internacional de Audiologia, realizado na cidade de Bauru SP, nos dias 14-17 de Abril de 2000. Artigo recebido em 08 de novembro de 2002. Artigo aceito em 07 de fevereiro de 2003.
} 


\section{INTRODUÇÃO E REVISÃO DA LITERATURA}

Até a década de 70, o interesse pela sensibilidade do ouvido humano para sons de alta freqüência não era evidente, dadas as conclusões provenientes dos estudos da época, as quais revelaram que os precoces danos ocasionados ao sistema auditivo já poderiam ser identificados com a avaliação da freqüência de $8 \mathrm{kHz}^{1,2,3}$.

Entretanto, pesquisas mais recentes investigaram a possibilidade de se avaliar a sensibilidade auditiva num espectro de freqüência mais amplo (acima de $8 \mathrm{kHz}$ ) e trouxeram novas perspectivas relacionadas ao diagnóstico precoce de danos auditivos recentes, decorrentes de agentes etiológicos degenerativos, como envelhecimento e exposição a drogas ototóxicas e a intensidades elevadas de ruído.

Apesar de existirem equipamentos que possibilitam a realização da audiometria tonal nas altas freqüencias (8$20 \mathrm{kHz}$ ), tais pesquisas ainda não mostraram um consenso quanto à importância desses sons e abordaram a falta de fidelidade aos padrões de calibração, as limitações dos equipamentos, as metodologias empregadas e os resultados encontrados. Tais dúvidas demonstram a carência do conhecimento relacionado à normalidade e à patologia.

Os resultados encontrados até o presente momento, em indivíduos audiologicamente normais, mostram evidências da excelente sensibilidade auditiva para sons de alta freqüência em crianças ${ }^{4-7}$. Outros autores ${ }^{6,8-15}$, estudando adultos, evidenciaram uma tendência ao declínio da sensibilidade, com o avanço da idade.

A variabilidade entre sexos tem sido bastante abordada, principalmente em pesquisas relacionadas à população com algum tipo de deficiência auditiva, ou de faixa etária mais velha. De acordo com a literatura ${ }^{13,16}$, as mulheres têm demonstrado, freqüentemente, melhor sensibilidade auditiva do que os homens para sons de alta freqüência. Porém, em se tratando de uma população jovem e audiologicamente normal, os resultados das pesquisas geralmente não têm demonstrado diferenças significantes nessa comparação.

A dificuldade em encontrar resultados precisos levou ao surgimento de diversas técnicas de avaliação. Está claro que determinados procedimentos, criados com a finalidade de solucionar variabilidades inter e intra-indivíduo e variabilidades acústicas, pertinentes à avaliação de alta freqüência, até seriam viáveis como fins científicos, mas não serviriam como procedimento para o diagnóstico precoce na prática clínica, onde as avaliações e técnicas utilizadas devem apresentar boa confiabilidade nos resultados e facilidade de aplicação.

\section{Dificuldades relacionadas à avaliação audiológica com sons de alta freqüência.}

Apesar da diminuição da sensibilidade auditiva em função da freqüência ser uma tendência comum, como já foi citado, alguns autores ${ }^{6,17,18}$ já alertavam que a instabilidade nos resultados pode ser provocada não somente por fatores intrínsecos ao indivíduo, como também por aqueles relacionados à metodologia empregada. Assim, destacaram que os próprios critérios que caracterizam a população selecionada, as técnicas empregadas na calibração dos equipamentos e na realização dos exames, as características do ambiente de teste e os próprios critérios de resposta do indivíduo podem ser fatores agravantes para o aumento na variabilidade dos resultados entre indivíduos de um mesmo grupo etário, de uma mesma faixa etária ou de um mesmo indivíduo, em diferentes exames. Os autores aconselharam, que cuidados especiais precisam ser tomados na comparação dos achados, em estudos relacionados à normalidade e à patologia.

A dificuldade no posicionamento dos fones ao ouvido deve-se à presença das variabilidades acústicas e biológicas e ocorre porque quando $1 / 4$ do comprimento de onda se aproxima do comprimento do conduto auditivo externo (CAE), ressonâncias e ondas estacionárias são produzidas, alterando o sinal-teste inicial e dificultando a precisa medida do nível de pressão sonora junto à membrana timpânica.

Isto freqüentemente acontece com sons de freqüência acima de $3 \mathrm{kHz}$. Entretanto, acima de $15 \mathrm{kHz}$, a metade do comprimento de onda já é suficiente para produzir tais ressonâncias nas dimensões transversais do CAE. Desta forma, não existe por todo o meato, uma frente de onda uniforme com estabilidade do nível de pressão sonora, até a membrana timpânica. Variações de aproximadamente $15-20 \mathrm{~dB}$ não são raras nesses casos e podem ser resultantes de mínimas modificações no posicionamento dos fones ao ouvido.

Adicionando-se o fato de que a membrana timpânica está posicionada de forma angular ao final do CAE e em diferentes graus para cada indivíduo e levando-se em consideração a geometria do meato, a distribuição da onda sonora torna-se ainda mais irregular ${ }^{1-3,19-21}$.

Assim, faz-se necessário obter informações atuais e consistentes a respeito do comportamento desses limiares em indivíduos audiologicamente normais e da confiabilidade do exame, desde a detecção, até o monitoramento da deficiência auditiva, principalmente em patologias sensório neurais, que geralmente mostram seu início a partir das freqüências mais altas.

A proposta desta pesquisa foi observar os limiares auditivos nas altas freqüências, em indivíduos audiologicamente normais, bem como analisar variabilidades acústicas inter e intra-indivíduo, que podem provocar interferências nos resultados do teste.

\section{MATERIAL E MÉTODO}

O presente trabalho foi realizado no Centro de Distúrbios da Audição, Linguagem e Visão do Hospital de Reabilitação de Anomalias Craniofaciais (CEDALVI-HRAC- 
USP), sendo aprovado pelo Comitê de Ética em Pesquisa do mesmo Hospital.

Foram avaliados 50 indivíduos ( 24 do sexo masculino e 26 do feminino), na faixa etária compreendida entre 18 e 30 anos de idade. O processo de seleção constou de anamnese, inspeção otológica clínica, medidas de imitância acústica, pesquisa dos reflexos acústicos estapedianos, audiometria tonal convencional (ATL-Cv, 0,25-8kHz) e logoaudiometria, a fim de selecionar uma população que estivesse audiologicamente dentro dos padrões de normalidade.

Os critérios que definiram o padrão para as medidas de imitância acústica, para os reflexos acústicos estapedianos, para a ATL-Cv e para a logoaudiometria foram estabelecidos por Jerger ${ }^{21}$, Lacerda ${ }^{22}$ e ANSI S3.6. ${ }^{23}$, respectivamente.

O equipamento utilizado foi o audiômetro SD50 da marca SIEMENS, que emprega fones de ouvido HDA200 da marca SENNHEISER, com correções para Nível de Audição, de acordo com o Certificado 1.51-9493/92 e 14738/93 e padrões ANSI S3.6 $6^{23}$, Normas de Segurança IEC 645 e vibrador ósseo B71, ANSI $3.13^{24}$ e ISO $389^{25}$. Foram pesquisadas as freqüências de 9,10,11.2, 12.5, 14 e $16 \mathrm{kHz}$, sendo, esta última, o limite superior de freqüência do equipamento até o presente momento.

Para a pesquisa dos limiares audiométricos por via aérea, foi utilizada a técnica descendente, com intervalos de $10 \mathrm{~dB}$, até que o indivíduo não mais respondesse ao som. A partir dessa intensidade, a técnica ascendente foi utilizada com intervalos de $5 \mathrm{~dB}$, até que o indivíduo voltasse a ouvir. O limiar auditivo foi estabelecido em 50\% das repostas obtidas em tal intensidade.

Dois examinadores (A e B) foram responsáveis pela execução dos exames, a fim de analisar variabilidades intraindivíduo, num mesmo dia de testes. Os dois resultados foram comparados entre si.

Os fones de ouvido foram posicionados pelos próprios examinadores (examinador A, primeiramente), após uma breve orientação ao indivíduo sobre a tarefa a ser realizada durante o teste. Ao final da pesquisa dos limiares em todas as freqüências citadas, o próprio indivíduo retirou e reposicionou os fones ao ouvido e nova pesquisa de limiares foi realizada apenas nas freqüências de 9 e $16 \mathrm{kHz}$. Essa etapa foi incluída para averiguar a variabilidade de resultados, relatada na literatura, proveniente de qualquer modificação no posicionamento da fonte sonora ao ouvido (variabilidade acústica). Os resultados obtidos nas freqüências de $9 \mathrm{k} \mathrm{e} 16 \mathrm{kHz}, \mathrm{com}$ o posicionamento dos fones feito pelo examinador, foram comparados aos novos resultados, obtidos nas mesmas freqüências (9k e 16kHz), após o reposicionamento dos fones pelo próprio indivíduo.

Num outro dia, nova avaliação foi conduzida, com o examinador B iniciando as avaliações e seguindo o mesmo roteiro do primeiro dia, com o objetivo de analisar a variação intra-indivíduo, em dias diferentes de teste. Esse cuidado foi tomado tendo em vista que os fatores relacionados ao aprendizado e ao cansaço do indivíduo em teste poderiam interferir nos resultados finais.

Foram necessários dois dias para a conclusão dos testes em cada indivíduo. Os resultados obtidos pelo mesmo examinador (A ou B), em dias diferentes de teste, também foram comparados.

Desta forma, quatro audiogramas de alta freqüência foram obtidos de cada indivíduo, sendo quatro limiares nas freqüências de 10 a $14 \mathrm{kHz}$ e oito limiares nas freqüências de 9 e $16 \mathrm{kHz}$

A ordem de apresentação das freqüências, a escolha do ouvido-teste e a escolha das freqüências de reteste $(9 \mathrm{e}$ $16 \mathrm{kHz}$ ) foram feitas aleatoriamente para cada indivíduo, para que também, nesse momento, fatores como o aprendizado e o cansaço não interferissem nos resultados da pesquisa. No segundo dia de testes, os exames foram realizados na mesma sala acústica, aproximadamente à mesma hora do primeiro dia e com a mesma seqüência de freqüências estabelecida no dia anterior.

A AT-Cv e a logoaudiometria foram realizadas após a pesquisa dos limiares de alta freqüência, prevenindo o possível cansaço e a falta de atenção durante o teste da AT$\mathrm{AF}$, no primeiro dia.

Assim, tentou-se controlar variáveis pertinentes à rotina diária do indivíduo, como o estresse, a exposição ao ruído ambiental e de lazer, o tempo de atenção, o horário, o tempo de exame e a temperatura do ambiente, característicos daquele período do dia e outras variáveis que pudessem prejudicar a estabilidade dos resultados. O Quadro 1 mostra o roteiro de avaliações as quais passaram os indivíduos.

A Análise de Variância (ANOVA) foi o teste estatístico empregado na comparação dos resultados entre o sexo masculino e o feminino, entre o ouvido direito e o esquerdo e entre os exames realizados no mesmo dia em dias diferentes. O TEST-T, ou TESTE DE STUDENT, foi utilizado para comparar os resultados obtidos no teste e reteste das freqüências de 9 e $16 \mathrm{kHz}$.

\section{RESULTADOS}

As análises estatísticas dos dados permitiram traçar um perfil audiométrico da população estudada, bem como analisar as variáveis citadas.

$\mathrm{O}$ teste estatístico empregado nesta pesquisa (ANOVA, $\operatorname{com} \mathrm{f}=0,05$ e $\mathrm{p}=0,82$ ) não identificou diferenças significantes entre o sexo masculino e o feminino (Gráfico 1). Tais resultados entram em concordância com os de Hallmo et al. ${ }^{13}$ e Mattews et al. ${ }^{16}$.

Quanto à variabilidade interaural, o ouvido esquerdo (OE) apresentou melhor sensibilidade do que o direito (OD), na maioria das freqüências, como mostra o Gráfico 2. Porém, o teste estatístico ANOVA ( $\mathrm{f}=3,34 \mathrm{e} \mathrm{p}=0,07)$ não demonstrou diferenças significantes nessa análise. A ausência de diferenças 
Quadro 1. Seqüência do processo de avaliação

\begin{tabular}{|c|c|c|c|}
\hline \multicolumn{2}{|c|}{ Primeiro Dia } & \multicolumn{2}{|c|}{ Segundo Dia } \\
\hline Examinador $\mathrm{A}$ & Examinador $\mathrm{B}$ & Examinador $\mathrm{B}$ & Examinador $\mathrm{A}$ \\
\hline 1.Anamnese & 6.AT-AF & 10.Inspeção Otológica Clínica & 14.AT-AF \\
\hline 2.Inspeção Otológica Clínica & $\begin{array}{l}\text { 7. Reteste das freqüências } \\
\text { de } 9 \text { e } 16 \mathrm{kHz}\end{array}$ & 11.Timpamometria & $\begin{array}{l}\text { 15. Reteste das freqüências } \\
\text { de } 9 \text { e } 16 \mathrm{kHz}\end{array}$ \\
\hline 3.Medidas de Imitância Acústica & 8.AT-CV & 12.AT-AF & \\
\hline 4.AT-AF & 9.ATL-CV,SRT e IRF & $\begin{array}{l}\text { 13.Reteste das freqüências } \\
\text { de } 9 \text { e } 16 \mathrm{kHz}\end{array}$ & \\
\hline $\begin{array}{l}\text { 5.Reteste das freqüências } \\
\text { de } 9 \text { e } 16 \mathrm{kHz}\end{array}$ & & & \\
\hline
\end{tabular}

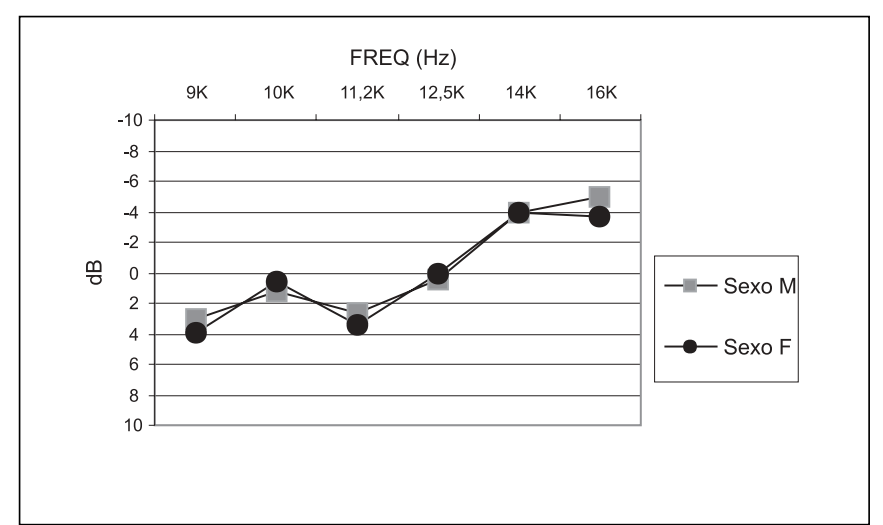

Gráfico 1. Média de limiares (dBNA) dos indivíduos do sexo masculino e do feminino, em todo o campo de freqüências

interaurais estatisticamente significantes foi notada ainda por Frank e Dreisbach ${ }^{11}$, Hallmo et al. ${ }^{13}$, Mattews et al. ${ }^{16}$.

Pôde-se, então, agrupar toda a população, a fim de estabelecer os limiares audiométricos para todo o campo de freqüências analisado. (Gráfico 3 e Tabela 1). A sensibilidade auditiva melhorou em função do aumento da freqüência e a média dos limiares ficou em torno de $3 \mathrm{~dB}$ em $9 \mathrm{kHz}$, chegando a atingir $-4 \mathrm{~dB}$ em $16 \mathrm{kHz}$.

É preciso ressaltar que o presente trabalho beneficiouse do equipamento SD50, o qual utiliza a norma ANSI S $3.6^{23}$ e ISO $389^{25}$ para a emissão de sons de alta freqüência, calibrados em níveis de audição (dBNA). Os demais trabalhos relatados na revisão de literatura analisaram os limiares em níveis de pressão sonora (dBNPS), de acordo com a norma ANSI S $3.6^{26}$, encontrando, em sua maioria, piora da acuidade auditiva, sugerindo um decréscimo na sensibilidade auditiva em função do aumento da freqüência.

Essa característica, encontrada nas pesquisas anteriores,

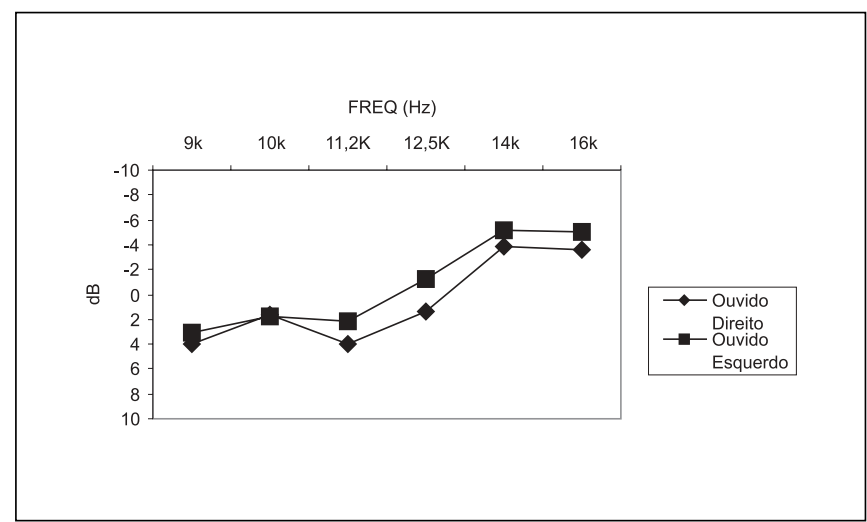

Gráfico 2. Média de limiares (dBNA) para o OD e para o OE em todo o campo de freqüências

é justificada pelo fato do ouvido humano necessitar de maior energia sonora para conseguir detectar os tons de alta freqüência, o que demonstra o comportamento fisiológico da cóclea aos tons do teste.

A calibração do equipamento SD50, se adaptada para a avaliação da acuidade auditiva em níveis de audição (dBNA), mostrará, logicamente, configuração audiométrica plana, em função da correção realizada na intensidade dos tons do teste, da mesma forma como ocorre com os audiômetros destinados à avaliação audiométrica convencional.

Pelo fato dessas correções estarem presentes no equipamento utilizado neste trabalho (dBNPS para dBNA), não foi possível realizar comparações entre limiares brutos.

Apesar de não ser proposta deste trabalho a criação de um padrão de normalidade para o campo audiométrico da altas freqüências, observou-se que as médias não ultrapassaram valores de +5 e $-5 \mathrm{dBNA}$ em toda a faixa de freqüência. 


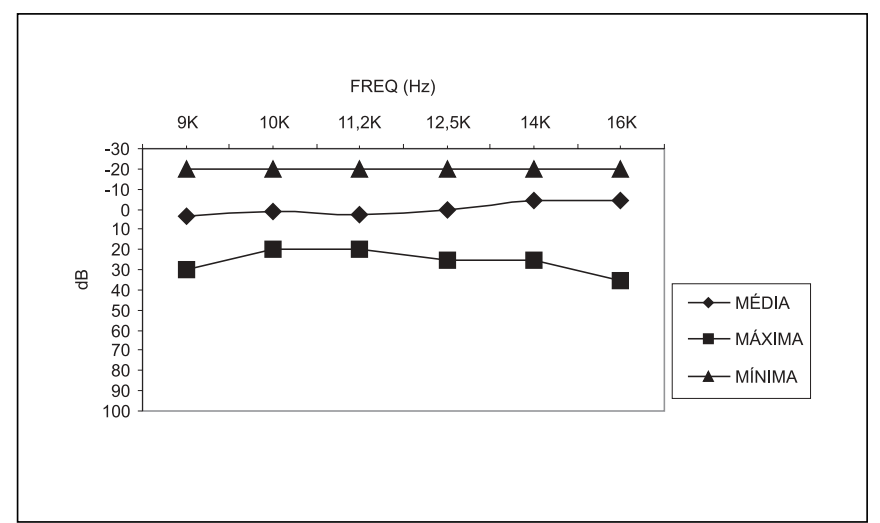

Gráfico 3. Média, mínimos e máximos (dBNA) para todo o grupo, em todo o campo de freqüências

Tabela 1. Valores de média, mínimos e máximos (dBNA) para toda a população, em todo o campo de freqüências analisado

\begin{tabular}{lcccccc}
\hline & $9 \mathrm{kHz}$ & $10 \mathrm{kHz}$ & $11.2 \mathrm{kHz}$ & $12.5 \mathrm{kHz}$ & $14 \mathrm{kHz}$ & $16 \mathrm{kHz}$ \\
\hline MÉDIA & 3,54 & 1,04 & 3,06 & 0,56 & $-4,55$ & $-4,35$ \\
MÍNIMO & -20 & -20 & -20 & -20 & -20 & -20 \\
MÁXIMO & 30 & 20 & 20 & 25 & 25 & 35 \\
\hline
\end{tabular}

\section{Variabilidades que interferem nos resultados}

Feghali e Bernstein ${ }^{3}$, Stelmachowicz et al. ${ }^{20}$, Filipo et al. ${ }^{27}$, alertaram para a ocorrência das variabilidades acústicas na pesquisa dos limares de alta freqüência, já citadas anteriormente. Segundo os autores, quando presentes, elas podem provocar interferência nas análises inter e intraindivíduos, não proporcionando repetibilidade dos resultados e nem boa aceitabilidade clínica.

Ao induzir tal variabilidade na pesquisa dos limiares, $\mathrm{O}$ presente trabalho não encontrou diferenças estatisticamente significantes nos resultados. Essa análise, como já foi visto no material e método, foi feita por meio do teste-reteste das freqüências de $9 \mathrm{k}$ e $16 \mathrm{kHz}$, com os resultados obtidos antes e após o reposicionamento dos fones. Neste ponto, o presente trabalho discorda dos autores citados (Gráficos 4 e 5).

A instabilidade dos resultados, proveniente da variabilidade intra-indivíduo, geralmente está associada à própria variabilidade acústica, há pouco discutida, ou a fatores próprios do indivíduo. As modificações na posição da fonte sonora, o cansaço, a atenção dispensada ao exame e o aprendizado podem não promover a estabilidade de resultados necessária para que o monitoramento audiométrico seja eficiente em pacientes com história de exposição a qualquer agente agressor ao sistema auditivo.

Nesta pesquisa, a análise da variabilidade intraindivíduo, realizada com os resultados de dois exames do

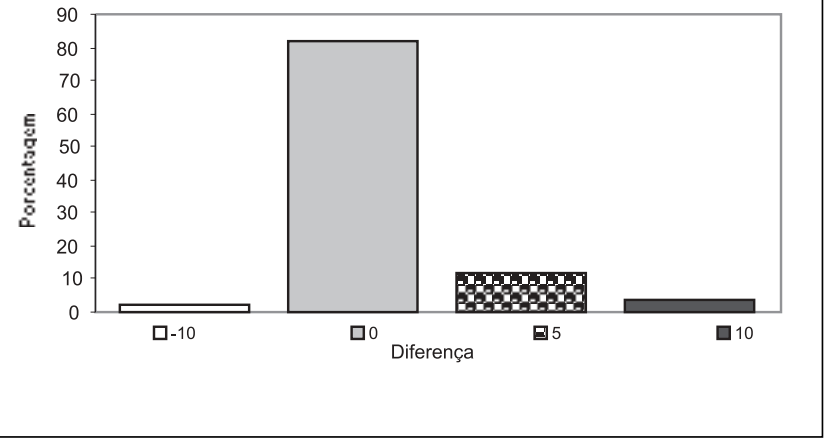

Gráfico 4. Distribuição da diferença (dBNA), entre teste e reteste para a freqüência de $9 \mathrm{khz}$

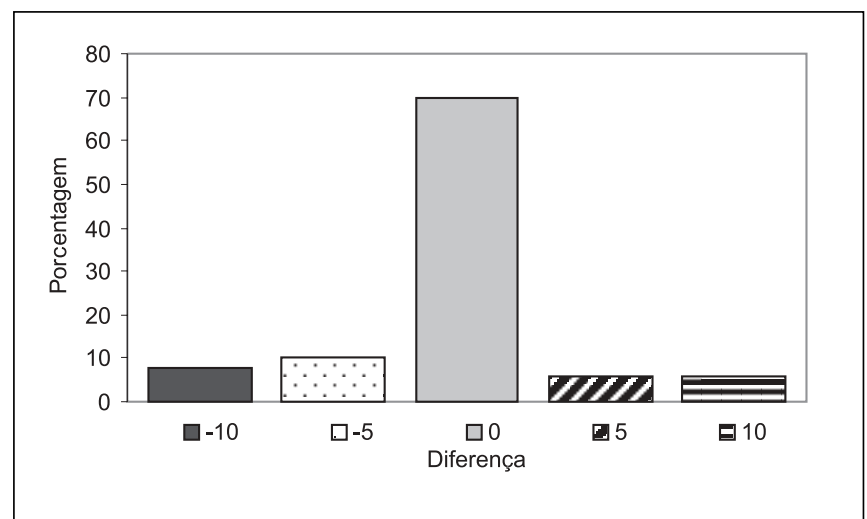

Gráfico 5. Distribuição da diferença (dBNA), entre teste e reteste da freqüência de $16 \mathrm{khz}$

mesmo indivíduo, num mesmo dia, não foi relevante. A análise (ANOVA, $\operatorname{com} \mathrm{f}=1,32$ e $\mathrm{p}=0,25$ ) não se mostrou estatisticamente significante (Gráficos 6) estando, portanto, de acordo com os trabalhos de Feghali e Bernstein ${ }^{3}$, Frank e Dreisbach ${ }^{11}$, Hallmo et al. ${ }^{13}$, Mattews et al. ${ }^{16}$, Stelmachowicz et al. ${ }^{20}$.

Já a variabilidade intra-indivíduo, relacionada à mudança nos resultados dos exames de um mesmo indivíduo, realizados em dias diferentes, mostrou-se importante nesta pesquisa. A análise dos dados sugeriram que, muito provavelmente, os efeitos de prática com o exame podem ter beneficiado os resultados no segundo dia de teste, já que as diferenças foram estatisticamente significantes e ficaram em torno de $+/-5 \mathrm{~dB}$ nesse dia. (Gráfico 7).

Os resultados encontrados por Frank e Dreisbach ${ }^{11}$, Mattews et al. ${ }^{16}$ mostraram diferenças de até +/-10dB entre exames. Os achados desses autores, associados aos de Hallmo et al. ${ }^{13}$, não estão de acordo com os da presente pesquisa, pois as diferenças não foram estatisticamente significantes. 


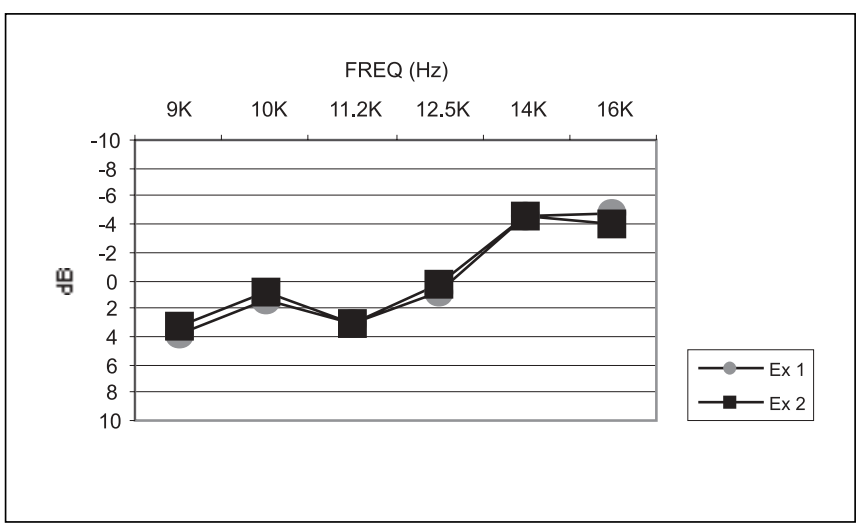

Gráfico 6. Média dos limiares (dBNA), nos exames realizados num mesmo dia, em todo o campo de freqüências

Nesta pesquisa, o aumento da variabilidade interindivíduos ocorreu em função do aumento da freqüência, sendo mais nítida acima de $12 \mathrm{kHz}$. Tal característica também foi discutida e evidenciada nos estudos de Feghali e Bernstein $^{3}$, Schechter et al. ${ }^{6}$, Fausti et al. ${ }^{9}$, Hallmo et al. ${ }^{13}$.

Vale a pena ressaltar que nos estudos citados foram utilizadas diferentes metodologias, como calibração dos equipamentos, fones de ouvido, técnica para a pesquisa de limiares, entre outros e, apesar disso, a conclusão sobre essa variação de limiares foi semelhante, denotando a marcante presença das variabilidades biológicas interferindo na comparação dos resultados entre os indivíduos.

Se cruzarmos essa informação com a análise da variabilidade intra-indivíduo, relacionada aos exames realizados em dias diferentes e com as médias dos limiares, que foram melhores no segundo dia, pode-se concluir que um treino prévio à avaliação de alta freqüência deve ser de grande valor, levando em consideração que o indivíduo em teste pode familiarizar-se com o exame antes de iniciá-lo efetivamente. Se esse treino puder ser realizado com as freqüências mais altas, principalmente acima de $12 \mathrm{kHz}$ (onde a variabilidade interindivíduos e as diferenças interaurais foram mais acentuadas), talvez o resultado final possa revelar, com mais precisão, a realidade sobre os limiares auditivos, em função do exame ser realizado com maior consistência, atenção e segurança por parte do indivíduo.

\section{CONCLUSÃO}

De acordo com a análise dos resultados dos testes estatísticos empregados nesta pesquisa, pôde-se concluir que não existiram diferenças entre a sensibilidade auditiva de homens e de mulheres, para sons de alta freqüência, assim como entre os ouvidos direito e esquerdo. Isto pode sugerir um padrão de respostas semelhante para toda uma população que apresente características homogêneas quanto ao perfil audiológico e faixa etária.

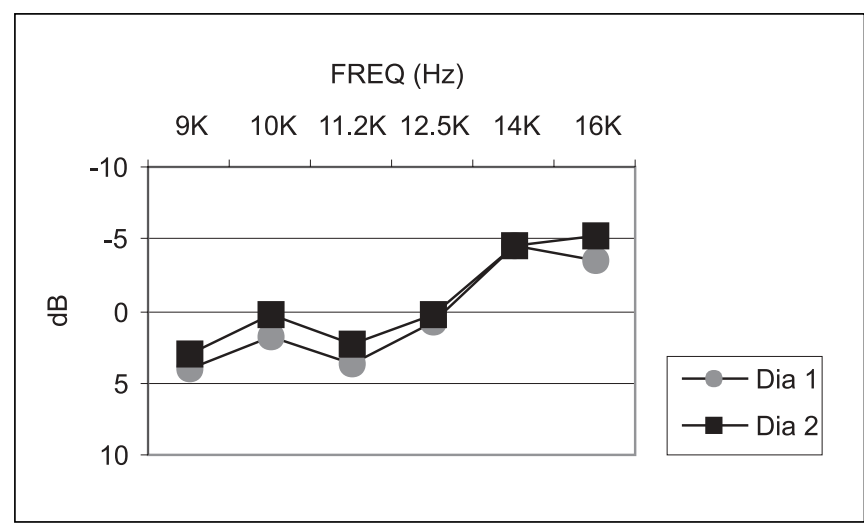

Gráfico 7. Média dos limiares (dBNA), nos exames realizados em dias diferentes, em todo o campo de freqüências

A instabilidade nos resultados entre os exames do mesmo indivíduo, levando a um monitoramento audiológico impreciso, poderá existir, caso ele não seja devidamente "treinado" para a realização do teste. As variações são decorrentes, muito provavelmente, da prática com o exame, já que as análises estatísticas não evidenciaram a importância de qualquer outra variabilidade estudada nesta pesquisa.

O treino citado nada mais seria do que a familiarização do indivíduo com os tons do teste. As análises do DP e das diferenças interaurais sugerem que esse treino deva ser realizado preferencialmente com as freqüências mais altas do campo audiométrico, ou seja, acima de $12 \mathrm{kHz}$, em função da maior variabilidade de resultados ter ocorrido na região dessa freqüência.

As variabilidades biológicas sempre existirão, interferindo nos resultados dos testes, sejam eles realizados com qualquer tipo de metodologia empregada. Elas são intrínsecas ao indivíduo e difíceis de controlar quando a metodologia está voltada à rotina clínica. No monitoramento audiológico das altas freqüências, os exames devem ser comparados individualmente e não entre indivíduos.

Apesar de não ser proposta deste trabalho a criação de um padrão de normalidade para o campo audiométrico de altas freqüências em indivíduos normais e de ocorrer variação interindivíduos, pôde-se observar a linearidade dos limiares auditivos, observada por meio das médias em cada freqüência analisada, permanecendo entre +5 e $-5 \mathrm{dBNA}$.

\section{AGRADECIMENTOS}

À Pontifícia Universidade Católica de São Paulo, ao Hospital de Reabilitação de Anomalias Craniofaciais - USP, ao Centro de Distúrbios da Audição Linguagem e Visão, ao Centro de Pesquisas Audiológicas, à FUNCRAF e à CAPES/ DS, pela disponibilidade em realizar este estudo.

Às Fonoaudiólogas Fabiana Velludo Bighetti e Isabela Monteiro de Castro, pela colaboração na avaliação dos indivíduos. 


\section{REFERÊNCIAS BIBLIOGRÁFICAS}

1. Tonndorf J, Kurman B. High frequency audiometry. Ann Otol Rhinol Laryngol 1984;93: 576-582.

2. Stelmachowicz PG, Beauchaine KA, Kalberer A, Langer T, Jesteadt $\mathrm{W}$. The reliability of auditory thresholds in the 8- to $20-\mathrm{kHz}$ range using a prototype audiometer. J Acoust Soc Am 1988;83:1528-1535.

3. Feghali JG, Bernstein RS. A new approach to serial monitoring of ultra-high frequency hearing. Laryngoscope 1991;101:825-829.

4. Zislis T. Relation of high frequency thresholds to age and sex. J Aud Res 1966;6:189-198.

5. Schneider B, Trehub SE, Bull D. High-frequency sensitivity in infants. Science 1980;207:1003-1004.

6. Schechter MA, Fausti SA, Rappaport BZ, Frey RH. Age categorization of high-frequency auditory threshold data. J Acoust Soc Am 1986;79: 767-771.

7. Trehub SE, Schneider BA, Morrongiello BA, Thorpe LA Developmental changes in high-frequency sensitivity. Audiology 1989;28:241-249.

8. Osterhammel D. High-frequency thresholds using a quasi-freefield technique. Scand Audiol 1978;7:27-30.

9. Fausti SA, Frey RH, Erickson DA, Rappaport BZ, Cleary EJ, Brummett RE. A system for evaluating auditory function from 8000-20000Hz. J Acoust Soc Am 1979;66:1713-1718.

10. Green DM, Kidd Jr. G, Stevens KN. High-frequency audiometric assesment of a young adult population. J Acoust Soc Am 1987;81:485-494.

11. Frank T, Dreisbach LE. Repeatibility of high-frequency thresholds. Ear Hear 1991;12:294-295.

12. Löppönen H, Sorri M. High-frequency air-conduction and eletric bone-conduction audiometry. Comparison of two methods. Scand Audiol 1991;20:175-180

13. Hallmo P, Borchgrevink HM, Mair IWS. Extended high-frequency thresholds in noise-induced hearing loss. Scand Audiol 1995;24:47-52.

14. Fouquet ML. Limiares de audibilidade nas freqüências ultra-altas de 9 a $18 \mathrm{kHz}$ em adultos de 18 a 30 anos [Especialização]. São Paulo: Universidade Federal de São Paulo, Escola Paulista de Medicina; 1997.
15. Azevedo LL. Estudo dos limiares de audibilidade nas altas freqüências em indivíduos de 12 a 15 anos com audição normal [Especialização] São Paulo: Universidade Federal de São Paulo, Escola Paulista de Medicina; 1997.

16. Mattews LJ, Lee FS, Mills JH, Dubno JR. Extended high-frequency thresholds in older adults. J Speech Hear Res 1997;40:208-214.

17. Fletcher JL. Reliability of high-frequency thresholds. J Aud Res 1965;5:133-137.

18. Northern JL, Downs MP, Rudmose W, Glorig A, Fletcher JL. Recomended high-frequency audiometry threshold level (800018000Hz). J Acoust Soc Am 1971:52:585-595.

19. Stevens KN, Berkovitz R, Kidd G, Green DM. Calibration of ear canals for audiometry at high frequencies. J Acoust Soc Am 1987;81:470-484.

20. Stelmachowicz PG, Beauchaine KA, Kalberer A, Jesteadt W. Normative thresholds in the $8-$ to $20-\mathrm{kHz}$ range as function of age. J Acoust Soc Am 1989;86:1384-1391.

21. Stelmachowicz PG, Beauchaine KA, Kalberer A, Kelly WJ, Jesteadt W. High-frequency audiometry: test reliability and procedural considerations. J Acoust Soc Am 1989;85:879-887.

22. Jerger $S$, Jerger J. Alterações auditivas. Um manual para avaliação clínica. Rio de Janeiro: Livraria Atheneu; 1989.

23. Lacerda AP. Audilogia clínica. Rio de Janeiro: Guanabara Koogan; 1976.

24. American National Standard Institute. American National Stadard Specification for Audiometers (ANSI S3.6). New York: ANSI; 1989.

25. American National Standard Institute. American National Stadard Specification for Audiometers (ANSI 3.13). New York: ANSI; 1972.

26. International Organization for Standardization. ISO 389 ADDA Acoustics. Standard Reference zero for the calibration of puretone audiometers. Geneva: International Organization for Standardization; 1983.

27. American National Standard Institute. American National Stadard Specification for Audiometers (ANSI 3.6). New York: ANSI; 1969

28. Filipo R, De Seta E, Bertoli BA. High-frequency audiometry in juvenile diabetes. Adv Audiol 1985;3:106-111. 